Blume, S. O. P., Ben-Mrad, R., \& Sullivan, P. E. (2015). Modelling the capacitance of multi-layer conductorfacing interdigitated electrode structures. Sensors and Actuators B: Chemical, 213, 423-433. doi:10.1016/j.snb.2015.02.088

\title{
Modeling the capacitance of multi-layer conductor-facing interdigitated electrode structures
}

\author{
Steffen O.P. Blume, Ridha Ben-Mrad, Pierre E. Sullivan \\ Department of Mechanical and Industrial Engineering, University of Toronto, Canada
}

\begin{abstract}
Interdigitated electrode structures have applications in a myriad of fields and have become attractive for in-line electrochemical detection in lab-on-a-chip microsystems. Analytical models can replace complex and expensive numerical simulations to determine the capacitance of interdigitated electrode structures in cases where simple geometries can be assumed. Closed-form analytical expressions derived from Schwarz-Christoffel conformal mappings were used to determine the capacitance of multi-layer interdigitated electrode structures with an additional parallel continuous electrode. The partial-capacitance approach was reformulated to find the capacitance of multi-layer structures with non-monotonically changing permittivities. The analytical model was found to be in close agreement with finite-element simulations. The model is used for optimization of an insulated transducer for sensitive detection of surface processes.
\end{abstract}




\section{Introduction}

Interdigitated electrode structures have been employed across many fields, from acoustic sensing in telecommunications to nondestructive testing [1]. The development of lab-on-a-chip devices and the associated need to downscale instrumentation for on-chip analyte detection and manipulation has driven the need for innovation of miniaturized transducers. Microfabricated interdigitated electrode structures are widely used in microfluidic systems for in-line analytical bioelectrochemistry $[1,2,3,4,5,6,7]$ and dielectrophoretic particle control $[8,9,10,11,12]$.

To design for high sensitivity of the electrochemical transducer, it is important to estimate the electrical coupling between the electrode fingers of the interdigitated multi-electrode array. While numerical models can provide an accurate representation of the physical, chemical, and electrodynamic characteristics of an electrochemical cell, they put high computational costs on the design and optimization process. Therefore, inexpensive analytical solutions that can estimate the capacitive coupling between the electrode fingers can be useful as a priori estimates.

Such closed-form analytical expressions that determine electrodynamic characteristics of coplanar electrode structures can be derived from conformal mapping methods [13]. For instance, these have been applied to determine the cell constants of planar-interdigitated conductivity sensors [14], for optimization of an electrolyte conductivity sensor [15], characterization of coplanar electrodes in microchannels [16, 17], optimization for the detection of affinity binding of biomolecules [18], and to determine the electric field pattern of two-electrode structures in flow cytometer designs [19].

In many applications, interdigitated electrode fingers are embedded below multiple dielectric or conductive layers, that are assumed to be stratified with respect to the electrode plane. Igreja \& Dias [20, 21] demonstrated that conformal mappings and the partial capacitance method can be used to determine the capacitance and impedance of interdigital electrodes in multi-layered structures with semi-infinite domain boundaries above the electrodes. They discussed the effects of monotonically decreasing and increasing permittivity profiles of the dielectric layers above the interdigitated electrodes. They found that their results correlated considerably better with numerical finite-element simulations than models previously reported by Wu et al. [22] and Grevorgian [23].

The current research efforts have focused on the integration of interdigitated electrode structures into digital microfluidic (DMF) devices for in-line electrochemical detection. Comprehensive reviews of 
digital microfluidics can be found in $[24,25,26,27]$. When embedding interdigitated multi-electrode arrays within DMF devices, the domain boundary above the interdigitated electrode structure cannot be assumed at infinity, and in fact is terminated by a finite-domain continuous grounded top electrode. In addition, hydrophobic passivation layers that prevent fluid adhesion and electrode degradation result in non-monotonical permittivity profiles.

The works on analytical expressions for the electric- and magnetic field patterns in conductor-backed coplanar waveguides [28], as well as the effect of a ground plane on coplanar lines [29] both relate to the effect of an additional parallel ground electrode. However, these did not account for multiple (dielectric) layers between the coplanar electrodes and the additional parallel electrode nor did they take into consideration interdigitated electrode structures. Hence a model is needed to determine the capacitance of an interdigitated electrode structure facing a continuous top electrode with multiple dielectric and conductive layers in between. The model shall support preliminary characterization of such electrode structures prior to more in-depth and expensive numerical simulation techniques.

Presented here is an approach based on Schwarz-Christoffel conformal mappings (SCM) to find the capacitance of interdigitated multi-electrode arrays with an additional parallel continuous ground electrode. It is shown that that SCM-aided approach can be used for structures of the same type as discussed in $[20,21]$. In addition, an adaptation of the partial capacitance method for multi-layer structures is presented, and it is further suggested that it can be applied to structures in which the permittivity of the stratified layers above the electrode fingers is changing non-monotonically. 


\section{Methods}

A sketch of a typical coplanar interdigitated electrode structure is shown in Figure 1. The structure has two interlocked comb-like electrode finger arrays to create alternating tiers of electrodes connected to two electrical terminals $(\phi=+V$ and $\phi=-V)$. The electrode fingers are assumed to have the same width $\mathrm{w}$ throughout and are separated by an inter-electrode gap $g$. The length $L$ is sufficiently large, such that three-dimensional fringing field effects near the electrode finger ends are negligible. The capacitance of a

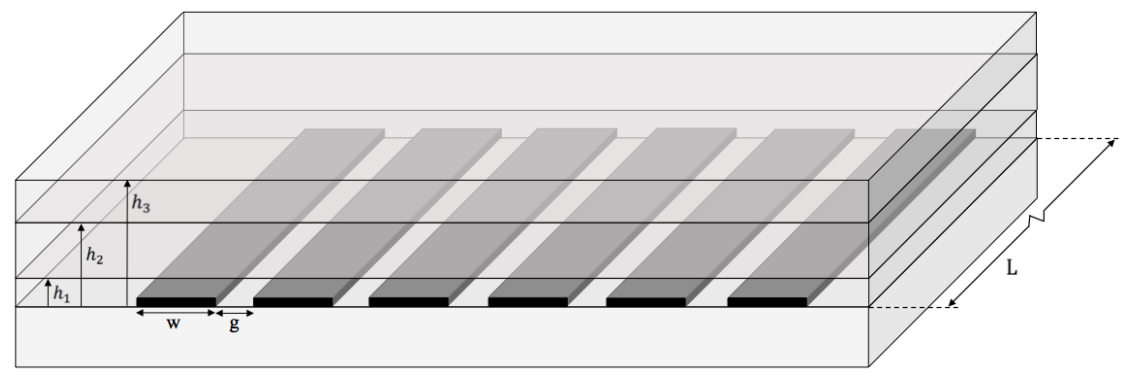

(a) Isometric view.

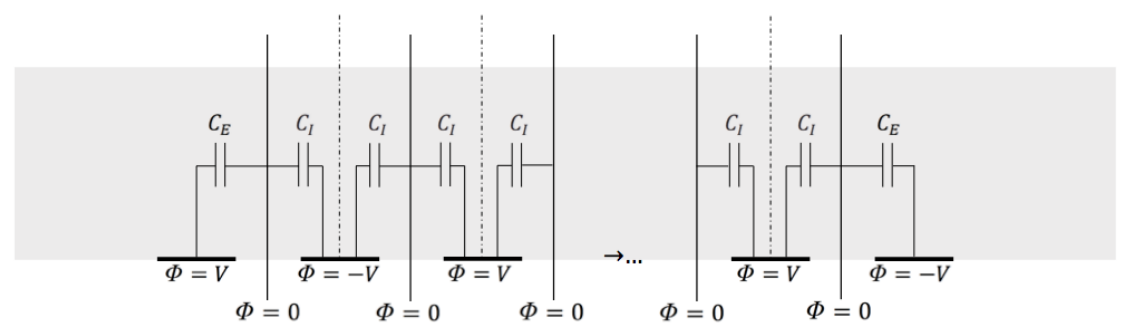

(b) Cross-section.

Figure 1 Example of an interdigitated electrode structure. (a) Example showing six electrode fingers, three top layers, and one bottom layer; (b) Capacitive coupling between unit cells.

multi-electrode structure with any even number of electrodes $N$, where $N \geq 4$, can be determined from network analysis of concatenated individual unit cells [20],

$$
C=(N-3) \frac{C_{I}}{2}+2 \frac{C_{I} C_{E}}{C_{I}+C_{E}}
$$

where $C_{I}$ is the capacitance of an interior unit cell, and $C_{E}$ is the capacitance of an exterior unit cell (Figure 1(b)). Accordingly, the capacitance of a two-electrode structure becomes $C=\frac{C_{E}}{2}$. How to determine unit cell capacitance is explained in coming paragraphs, and can be expressed in terms of two non-dimensional 
parameters: the metalization ratio, $\eta[20]$, and the height-to-width ratio, $r$, defined by

$$
\eta=\frac{\mathrm{w}}{\mathrm{w}+g}
$$

and

$$
r=\frac{2 h}{\mathrm{w}+g}
$$

where $h$ is the height of a layer above the electrode fingers. Moreover, the total capacitance including the layers below and above the interdigitated electrode structure is determined by adding the capacitance of the lower and upper half space.

\section{The partial capacitance method}

The interdigitated multi-electrode array is embedded below stratified layers, as shown in Figure 2 . The material is isotropic within each layer and only varies across layer interfaces in the vertical direction. The boundary conditions are specified according to the partial capacitance method and define the exterior and interior unit cells' geometry [20,21]. The partial capacitance method can be used to estimate the capacitance of a coplanar electrode structure embedded in a multi-layer assembly of dielectric materials with varying permittivity using either the parallel partial capacitance (PPC) or series partial capacitance (SPC) solution [30] based on [29, 31, 32, 23, 33]. The multi-layered interdigitated electrode structure in

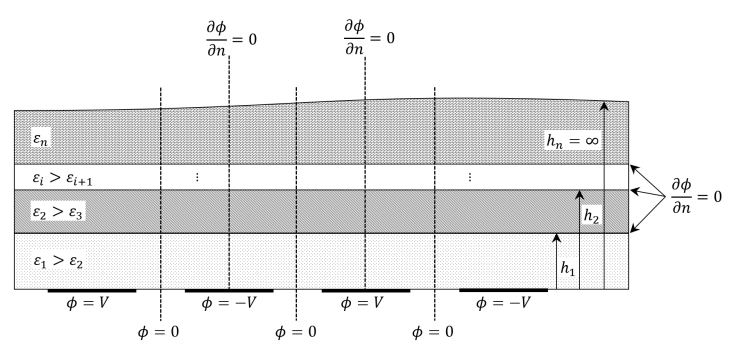

(a) $P P C$

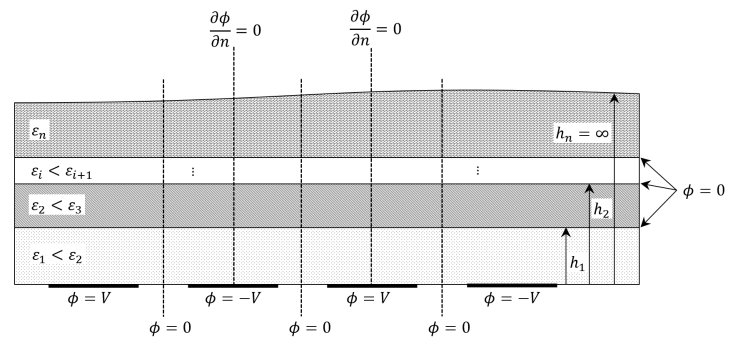

(b) $S P C$

Figure 2 Schematics of multi-layered interdigitated electrode structures in open configurations. This example structure consists of four electrode fingers. (a) The parallel partial capacitance (PPC) method is used for structures with decreasing permittivity. (b) The series partial capacitance (SPC) method is used for structures with increasing permittivity.

Figure 2(a) applies to the PPC method noting that the permittivity is decreasing across each layer interface away from the electrode plane. The reduction in permittivity from one layer to the next is assumed to 
act as an electric field barrier. The layers are cascaded in a parallel-type configuration, and Neumann boundary conditions (i.e., magnetic walls with $\frac{\partial \phi}{\partial n}=0$ ) are assumed at the layer interfaces. For the PPC method, the capacitance of a unit cell (interior or exterior) with $n$ layers is

$$
C_{\text {cell }}=\sum_{i=1}^{n-1}\left(\varepsilon_{r, i}-\varepsilon_{r, i+1}\right) C_{i}^{\prime}+\varepsilon_{r, n} C_{n}^{\prime}
$$

where $\varepsilon_{r, i}$ is the relative permittivity of the $i$ th layer, and $C_{i}^{\prime}$ is given by $C_{i}^{\prime}=\varepsilon_{0} L \kappa_{c e l l}^{c}\left(\eta, r_{i}\right)$. The cell constant $\kappa_{c e l l}^{c}$ is defined by the cross-sectional geometry (2-D) and boundary conditions of the unit cell, ignoring three-dimensional fringing field effects near the ends of the electrode fingers. The superscript $c$ indicates that the the unit cells are considered as capacitive elements ${ }^{1}$.

For cases when the permittivity is monotonically increasing and the electric field is more strongly guided away from the electrode plane, the layers are assumed to be coupled in series, yielding the SPC method (Figure 2(b)). It assumes Dirichlet boundary conditions (i.e., electric walls with $\phi=0$ ) at the layer interfaces, and the total capacitance of the unit cell becomes

$$
\frac{1}{C_{c e l l}}=\sum_{i=1}^{n-1}\left(\frac{1}{\varepsilon_{r, i}}-\frac{1}{\varepsilon_{r, i+1}}\right) \frac{1}{C_{i}^{\prime}}+\frac{1}{\varepsilon_{r, n}} \frac{1}{C_{n}^{\prime}}
$$

Note that the numerical value of the Dirichlet boundary condition is obviously different in reality. Here, it serves the purpose of forcing the electric field field lines be incident perpendicularly with the layer interfaces, whereas the Neumann boundary condition for the PPC approach mandates that the electric field lines are parallel to the layer interfaces.

The cell capacitance is hence specified by the permittivity and cell constant of the individual layers. Although not indicated explicitly, the cell constants in the PPC expressions are generally different than the cell constants in the SPC expressions, even though the height of the layers may be the same. This is because the magnitude of the cell constant is also determined by the choice of boundary conditions, i.e. Neumann or Dirichlet boundary conditions. Typically for open configurations the topmost layer is assumed to extend towards infinity, such that $C_{n}^{\prime}=\varepsilon_{0} L \kappa_{\text {cell }}^{c}(\eta, \infty)$, for both the PPC and SPC approach.

\footnotetext{
${ }^{1}$ Typically the cell constant is given as the geometric proportionality factor relating the resistance and resistivity.
} 


\section{Reformulating the partial capacitance approach}

Neither the PPC nor the SPC approach are applicable to instances with arbitrarily alternating permittivity across layer interfaces. The interdigitated electrode structure developed in the current work considers layers that result in non-monotonically varying permittivity. The partial capacitance approach can be reformulated such that it is possible to approximate the capacitance for any structure with arbitrarily varying permittivities. Equations (4) and (5) can be rewritten more explicitly in terms of the cell constant $\kappa_{\text {cell }}^{c}$,

$$
\begin{aligned}
& \mathrm{PPC}: C_{\text {cell }}=L\left[\sum_{i=1}^{n-1}\left(\varepsilon_{i}-\varepsilon_{i+1}\right) \kappa_{\text {cell }}^{c}\left(\eta, r_{i}\right)+\varepsilon_{n} \kappa_{\text {cell }}^{c}\left(\eta, r_{n}\right)\right] \\
& \mathrm{SPC}: \frac{1}{C_{\text {cell }}}=\frac{1}{L}\left[\sum_{i=1}^{n-1}\left(\frac{1}{\varepsilon_{i}}-\frac{1}{\varepsilon_{i+1}}\right) \frac{1}{\kappa_{\text {cell }}^{c}\left(\eta, r_{i}\right)}+\frac{1}{\varepsilon_{n}} \frac{1}{\kappa_{\text {cell }}^{c}\left(\eta, r_{n}\right)}\right]
\end{aligned}
$$

where $L$ is the length of the electrode fingers, and $\varepsilon_{i}=\varepsilon_{0} \varepsilon_{r, i}$. From this an equivalent relative permittivity can be defined as

$$
\begin{aligned}
& \mathrm{PPC}: \quad \varepsilon_{e q, i}=\left(\varepsilon_{e q, i-1}-\varepsilon_{i+1}\right) \frac{\kappa_{c e l l}^{c}\left(\eta, r_{i}\right)}{\kappa_{c e l l}^{c}\left(\eta, r_{i+1}\right)}+\varepsilon_{i+1} \\
& \mathrm{SPC}: \quad \varepsilon_{e q, i}=\frac{\varepsilon_{e q, i-1} \varepsilon_{i+1} \kappa_{c e l l}^{c}\left(\eta, r_{i}\right)}{\kappa_{\text {cell }}^{c}\left(\eta, r_{i+1}\right)\left(\varepsilon_{i+1}-\varepsilon_{e q, i-1}\right)+\varepsilon_{e q, i-1} \kappa_{c e l l}^{c}\left(\eta, r_{i}\right)}
\end{aligned}
$$

For $i=1, \varepsilon_{e q, i-1}=\varepsilon_{e q, 0}=\varepsilon_{1}$. Equation (7) combines two adjacent layers (layer $i$ and $i+1$ ) into one with an equivalent permittivity $\varepsilon_{e q, i}$ and height $h_{i+1}$, as illustrated in Figure 3(a).

Determining the equivalent permittivity is repeated until the last layer is reached, and the total capacitance of the unit cell is

$$
C_{c e l l}=\varepsilon_{e q, n-1} L \kappa_{c e l l}^{c}(\eta, \infty)
$$

The cell constant $\kappa_{\text {cell }}^{c}(\eta, \infty)$ indicates that the height of the topmost layer is infinite. This is equivalent to implementing Equations (4) and (5). The strength of this method is, however, that it can be applied to layers with arbitrarily varying permittivity (see Figure3(b)). The decision whether to choose the PPC or $\mathrm{SPC}$ expression is made by comparing the permittivities $\varepsilon_{i}$ and $\varepsilon_{i+1}$.

\section{Partial capacitance method for closed configurations}

In many cases the top layer can be assumed to be semi-infinite, i.e. open configurations. For the current work, cases arise in which the top layer cannot be assumed to be semi-infinite. In fact, the assumed structure 


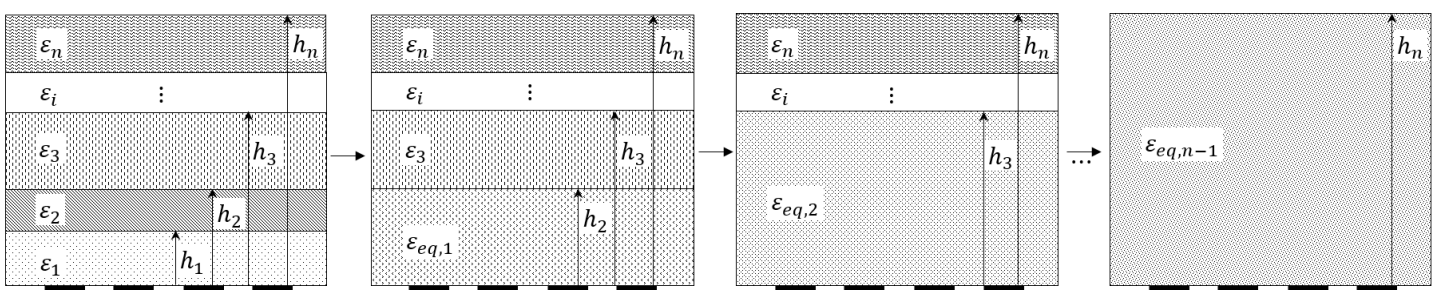

(a)

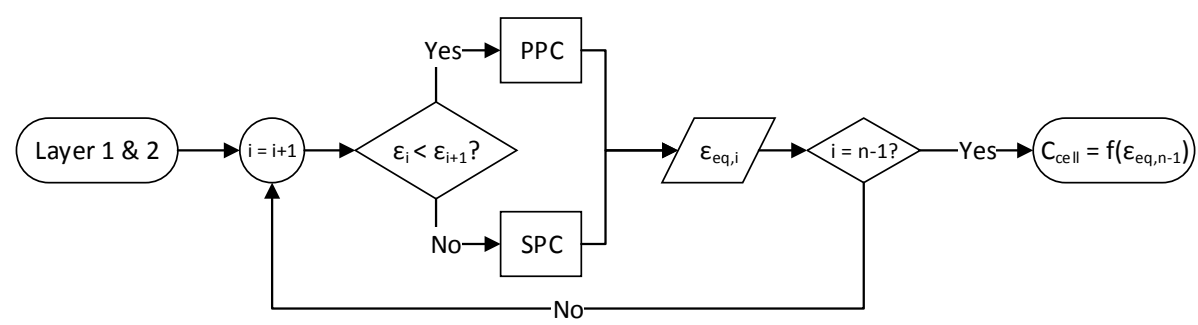

(b)

Figure 3 Adaptation of the partial capacitance method. (a) Schematic sequence illustrating the modified partial capacitance method; (b) Flow chart illustrating how to determine the equivalent relative permittivity for stratified layers with arbitrarily varying permittivity.

consists of multiple layers sandwiched between the multi-electrode array and a grounded continuous top plate. Therefore, a closed configuration with a geometry and unit cells as defined in Figure 4 is assumed.

To determine the capacitance for structures with decreasing permittivity away from the electrode plane, Figure 4(a), a solution would be to follow the procedure for open configurations. The equivalent permittivity is determined by assuming a PPC-type approach (Equation (7)) with Neumann boundary conditions at all layer interfaces, including the top surface of the topmost layer. Hence, determining the equivalent permittivity momentarily ignores that the top is electrically grounded and should be specified by a Dirichlet boundary condition. However, the Dirichlet boundary condition is reintroduced when evaluating the total cell capacitance according to

$$
C_{\text {cell }}=\varepsilon_{e q, n-1} L\left(\kappa_{\text {cell }}^{c}\left(\eta, r_{n}\right)\right)_{D B}
$$

Here, the subscript $D B$ indicates that the cell constant is determined by specifying a Dirichlet boundary condition at the topmost boundary. For the case of increasing permittivity, Dirichlet boundary conditions are assumed at the layer interfaces, as shown in Figure 4(b). The total capacitance is determined in a 


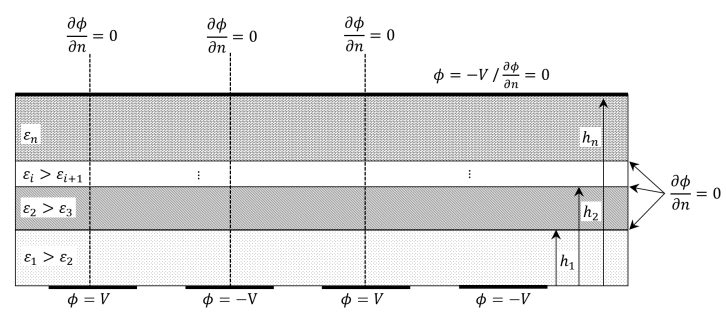

(a) $P P C$

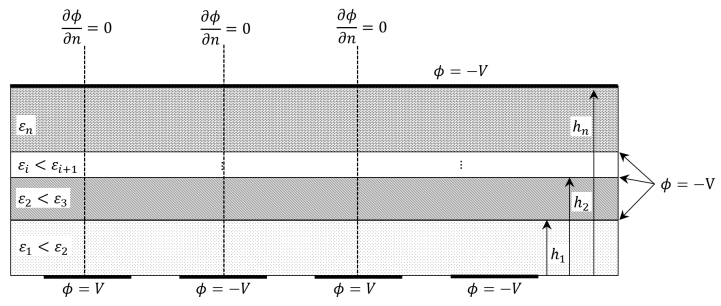

(b) $S P C$

Figure 4 Schematic diagrams of multi-layered interdigitated electrode structures in closed configurations. The topmost layer is constrained by a grounded electrode, such that the height $h_{n}$ is finite. (a) The parallel partial capacitance (PPC) method is used for structures with decreasing permittivity. (b) The series partial capacitance (SPC) method is used for structures with increasing permittivity.

similar fashion as in the SPC approach for open configurations (Equation (7)). In consecutive steps, an equivalent permittivity is determined until the total capacitance can be expressed by Equation (9). For non-monotonically changing permittivities, the procedure outlined previously for open configurations is still applicable. Additionally, for the leftmost exterior electrode unit cell, the change in the permittivity is disregarded, and SPC solution is used throughout.

\section{Results}

For a coplanar electrode structure, the cell constant is determined by transforming the physical space of the coplanar electrode structure into a model space in which the electrodes are parallel and the electric field lines are uniformly aligned, mimicking an ideal parallel plate capacitor. The resulting boundary value problem in the model domain can be easily solved. Backwards transformations are then used to determine electric field characteristics in the physical domain. The cell constant associated with the model space is equal to the cell constant of the physical space, since the ratio associated with the cell constant as well as Laplace's equation are invariant under conformal mapping [34].

By using Schwarz-Christoffel conformal mapping (SCM), the vertices and interior of the polygons associated with the unit cells of the interdigitated multi-electrode array are transformed into an intermediate domain, before being further mapped to the model domain. The use of two consecutive Schwarz-Christoffel conformal transformations for determining the cell constant has already been used by other groups largely interested in modeling the behavior of transmission lines, see [13]. Sun et al. have demonstrated an SCM-aided approach for flow cytometer designs [19] and the study of AC electrokinetics in interdigitated 
electrode arrays [35]. A detailed description of the method and derivation for the results presented here can be found in $[36]$.

\section{Crowding}

The expressions for the cell constants were implemented in MATLAB ${ }^{\circledR}$ (64-bit). Below a critical value of the height-to-width ratio, the cell constants could not be evaluated because of the long and thin regions defined by the geometry of the unit cells. Performing a transformation between the physical domain and the model domain involves consecutive mappings to intermediate domains. The vertices defined in the physical domain are mapped to prevertices in these intermediate domains. For narrow domains these prevertices become positioned extremely close together. The exact location between them cannot be distinguished because of crowding [37], i.e. limitations due to round-off errors and the double precision arithmetic of the computer. The onset of crowding results from the height of the physical domain being too small with respect to the remaining dimensions. For the models presented in this work the minimum height-to-width ratio that can be achieved was $r_{\text {min }} \approx 0.05$. However, as $r \rightarrow 0$ the cell constant can be approximated by analysis of the unit cell boundary conditions and geometry, given along with the following results.

\section{Cell constants for open configurations}

Figures 5(a) to 5(f) show the different types of unit cells that are considered for open configurations. Depending on whether the layers are assumed to be coupled in parallel or series, Neumann or Dirichlet boundary conditions are specified at the top surface, respectively. Tables 1 and 2 list the equations to determine the cell constants for exterior and interior unit cells, respectively. The expressions are based on the results of Blume [36]. While determined as given by Table 1, the solution for exterior electrodes with Neumann top boundaries agrees with the work presented by Sun et al. [19] on flow cytometer optimization.

Furthermore, the expressions given here and the ones presented in $[20,21]$ were found to return the same values of the cell constants for any combination of $\eta$ and $r$.

In Tables 1 and $2, K(k)$ is the complete elliptic integral of the first kind, and $k^{\prime}=\sqrt{1-k^{2}}$ is the complement of the elliptic modulus $k$. For the interior unit cells with finite height layers in Table 2 , the elliptic modulus is determined in terms of the Jacobi theta functions $\vartheta_{2}(0, q)$ and $\vartheta_{3}(0, q)$ according to

$$
k=\left(\frac{\vartheta_{2}(0, q)}{\vartheta_{3}(0, q)}\right)^{2}
$$


Table 1 Cell constants for exterior electrode unit cells with finite and infinite height layers in open configurations.

\begin{tabular}{|c|c|c|}
\hline $\begin{array}{l}\text { Finite height layer, } \\
\text { Dirichlet b.c. (SPC) }\end{array}$ & $\begin{array}{c}\text { Finite height layer, } \\
\text { Neumann b.c. (PPC) }\end{array}$ & Infinite height layer \\
\hline$\kappa_{\text {cell }}^{c}=\frac{K\left(k^{\prime}\right)}{K(k)}$ & $\kappa_{\text {cell }}^{c}=\frac{K\left(k^{\prime}\right)}{K(k)}$ & $\kappa_{\text {cell }}^{c}=\frac{K\left(k^{\prime}\right)}{K(k)}$ \\
\hline \multicolumn{2}{|c|}{ where } & where \\
\hline$k=\sqrt{\frac{t_{B}-1}{t_{A}-1}}$ & $k=\sqrt{\frac{t_{A}\left(t_{B}-1\right)}{t_{B}\left(t_{A}-1\right)}}$ & $k=\sqrt{t_{B}+1}$ \\
\hline \multicolumn{2}{|c|}{$t_{A}=\cosh ^{2}\left(\frac{\pi(1+\eta)}{2 r}\right)$} & $t_{B}=-\frac{4 \eta}{(\eta+1)^{2}}$ \\
\hline \multicolumn{2}{|c|}{$t_{B}=\cosh ^{2}\left(\frac{\pi(1-\eta)}{2 r}\right)$} & \\
\hline \multicolumn{2}{|c|}{$r \rightarrow 0$} & \\
\hline$\kappa_{\text {cell }}^{c}=\frac{2 \eta}{r}+1$ & $\kappa_{\text {cell }}^{c}=\frac{r}{1-\eta}$ & \\
\hline
\end{tabular}

Table 2 Cell constants for interior electrode unit cells with finite and infinite height layers in open configurations.

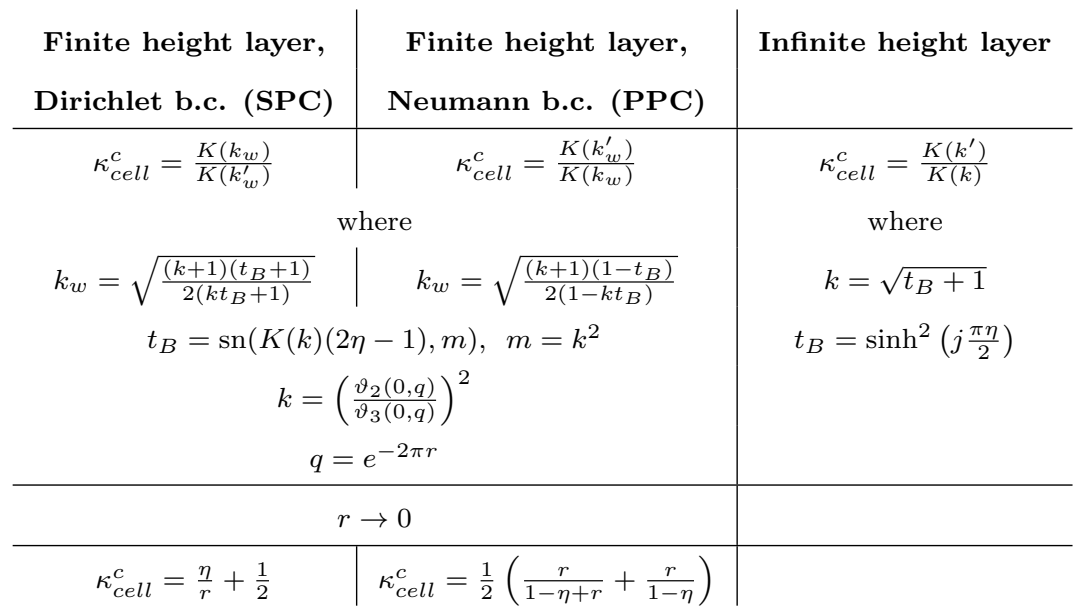


Alternatively, the elliptic modulus can be found from an approach suggested by Luther \& Otten [38] to determine $k$ based on a given value of the nome $q$. The solution for $k$ starts with an initial value

$$
k=4 \sqrt{q} \prod_{i \geq 1}\left(\frac{1+q^{2 i}}{1+q^{2 i-1}}\right)^{4}
$$

To arrive at a converged solution, a Newton-Raphson approach follows the initial guess, to minimize

$$
f(k)=e^{-\pi \frac{K\left(k^{\prime}\right)}{K(k)}}-q
$$

\section{Cell constants for closed configurations}

Figure 6 shows the types of unit cells considered for the closed configuration. The associated cell constants are presented in Table 3 based on the results of Blume [36].

The exterior unit cell is defined as a semi-infinite strip with boundary conditions specified as shown. The top surface of the interior unit cell is either defined by a Neumann or Dirichlet boundary condition. For a Neumann boundary condition the unit cell is symmetric. The equipotential symmetry line is indicated in Figure 6(b). Each of the two symmetric planes is equivalent to the unit cell considered for interior electrodes with Neumann boundary conditions in open configurations. The equivalent capacitance is half of the individual capacitance, such that in Table 3 the fraction $\frac{K\left(k_{w}^{\prime}\right)}{K\left(k_{w}\right)}$ is multiplied by $1 / 2$. For a Dirichlet boundary condition (i.e., $\Phi=-V$ ), the domain for the interior unit cell is simplified by assuming that the vertices $A$ and $F$ extend to infinity. A schematic of this domain is shown in Figure 7. Redefining the domain enables a straightforward derivation of the expression for the cell constant, although it has ramifications on accurately determining the capacitance of multi-finger structures. These issues are addressed later.

The total capacitance of the interdigitated electrode structure is expressed as [39]

$$
C=\frac{1}{4}\left[(N+2) C_{I}+(3 N-2) C_{E}\right]
$$

where the capacitance of the interior unit cells is $C_{I}$, and the cell capacitance of the exterior unit cell is $C_{E}$. 
Table 3 Cell constants for unit cells in closed configurations.

\begin{tabular}{|c|c|c|}
\hline $\begin{array}{l}\text { Exterior unit cell, } \\
\text { Dirichlet b.c. (SPC) }\end{array}$ & $\begin{array}{c}\text { Interior unit cell, } \\
\text { Neumann b.c. (PPC) }\end{array}$ & $\begin{array}{c}\text { Interior unit cell, } \\
\text { Dirichlet b.c. (PPC) }\end{array}$ \\
\hline $\begin{array}{c}\kappa_{\text {cell }}^{c}=\frac{K(k)}{K\left(k^{\prime}\right)} \\
\text { where } \\
k=\sqrt{\frac{t_{A}+1}{t_{A}}} \\
t_{A}=-\cosh ^{2}\left(\frac{\pi \eta}{2 r}\right) \\
r=\frac{2 h}{w+g} \text { with } g=0\end{array}$ & $\begin{array}{c}\kappa_{\text {cell }}^{c}=\frac{K\left(k_{w}^{\prime}\right)}{2 K\left(k_{w}\right)} \\
\text { where } \\
k_{w}=\sqrt{\frac{(k+1)\left(1-t_{B}\right)}{2\left(1-k t_{B}\right)}} \\
t_{B}=\operatorname{sn}(K(k)(2 \eta-1), m), \quad m=k^{2} \\
k=\left(\frac{\vartheta_{2}(0, q)}{\vartheta_{3}(0, q)}\right)^{2} \\
q=e^{-2 \pi r}\end{array}$ & $\begin{array}{c}\kappa_{\text {cell }}^{c}=\frac{K(k)}{K\left(k^{\prime}\right)} \\
\text { where } \\
k=\sqrt{\frac{\left(t_{C}-1\right) t_{B}}{\left(t_{B}-1\right) t_{C}}} \\
t_{B}=\cosh ^{2}\left(\frac{\pi(\eta-2)}{2 r}\right) \\
t_{C}=\cosh ^{2}\left(\frac{\pi \eta}{2 r}\right)\end{array}$ \\
\hline \multicolumn{3}{|c|}{$r \rightarrow 0$} \\
\hline$\kappa_{\text {cell }}^{c}=\frac{1}{r}$ & $\kappa_{\text {cell }}^{c}=\frac{1}{4}\left(\frac{r}{1-\eta+r}+\frac{r}{1-\eta}\right)$ & $\kappa_{\text {cell }}^{c}=\frac{\eta}{r}+\frac{1}{2}$ \\
\hline
\end{tabular}

\section{Discussion}

The analytical model was compared to two-dimensional finite element (FE) simulation data generated from COMSOL Multiphysics ${ }^{\circledR}$. The goal was to analyze the PPC and SPC approaches for a complete interdigitated electrode structure to investigate if scaling effects may occur for different numbers of electrodes. The analytical models described as well as prior work by other groups have certain limitations with regard to their application to electrochemical transducer structures.

The models are limited to simple interdigitated electrode structures, with two identical electrode arrays resulting in a straightforward two-dimensional cross-section. More complex structures in the horizontal plane cannot be modelled. However, these simple structures are widely found in literature.

Also, the electrode fingers need to be sufficiently long, such that fringing field effects near the electrode finger ends can be neglected. This is crucial for arrays with only a few fingers. It was shown in [36] that for two-electrode structures, the finger length $L$ should be approximately 10 times larger than the sum $(2 \mathrm{w}+g)$ to not incur significant errors.

In addition, the thickness of the electrodes is neglected. This assumption is potentially not appropriate where the thickness is within an order of magnitude of the lateral dimensions of the electrode fingers $(i . e$. , $\mathrm{w}$ and $g$ ). The research efforts in digital microfluidics that motivated this work deal with lateral dimensions in the order of microns, whereas the electrode thickness remains at least two orders of magnitude below this, allowing the electrode thickness to be ignored.

The models discussed here are limited to an even number of electrode fingers. However, they can be 
easily adjusted for odd numbers.

This work supports existing models without proposing additional limitations, and extends their applicability to interdigitated electrode structures with non-monotonical permittivity profiles and closed configurations. The value of these simple models is the small computational expense compared to numerical simulations and sufficient accuracy as a priori estimators for the capacitance of interdigitated electrode structures. Figure 8 compares finite element analysis (FEA) data to the SPC and PPC approach for a number of electrode and layer configurations. The assumed geometry is sketched in the insets in Figure 8. The graphs plot the capacitance per unit depth against the ratio between the permittivity of layer one and two. The relative permittivity of layer two was kept constant at 10, while the relative permittivity of layer one was varied from 1 to 100. Figures $8(\mathrm{a})$ and $8(\mathrm{~b})$ are distinguished by the height-to-width ratio of layer one, i.e. $r_{1}=0.1$ in Figure $8(\mathrm{a})$ and $r_{1}=0.5$ in Figure $8(\mathrm{~b})$. The graphs show that the PPC approach closely fits the numerical data for ratios above 1 , but fails to model the cell capacitance for $\varepsilon_{1} / \varepsilon_{2}$-ratios below 1. The reverse is true for the SPC approach.

The two methods work as expected: If the relative permittivity is decreasing away from the electrode plane, the PPC solution should be used, while for increasing permittivities the SPC solution is more appropriate. Therefore, the SPC and PPC method perform well in approximating the capacitance of interdigitated electrode structures in open configurations, independent of the tested number of electrodes.

The same analysis was done for interdigitated electrode structures in closed configurations. The structure again consists of two layers. The relative permittivity of layer one is varied from 1 to 100 , while the relative permittivity of layer two is held constant. Figure 9 (a) and 9(b) show the results for two different arrangements: One in which $r_{1}=0.1$, and one in which $r_{1}=0.5$. In both cases $r_{2}=\left(1.0+r_{1}\right)$ and $\eta=0.5$. Again, the analytical model output is close to the numerical simulation results, and both agree well with each other irrespective of the number of electrode fingers. As before, the SPC approach is valid for $\varepsilon_{1} / \varepsilon_{2} \leq 1$, and PPC approach is valid for $\varepsilon_{1} / \varepsilon_{2} \geq 1$.

The partial capacitance approach has been used for monotonically increasing or decreasing permittivities in stratified layers above coplanar electrodes. For this work, the attempt was to extend the approach to layers with arbitrarily varying permittivities. To test the procedure, a test structure consisting of three layers is used, and the analytical results are compared to FEA simulations.

Figure 10 compares the capacitance per unit depth of an open configuration obtained from FE analysis to the results from the analytical model for $N=2, N=12$, and $N=24$. The model structure corresponding 
to an open configuration is illustrated in the insets in Figure 10. The relative permittivities $\varepsilon_{1}$ and $\varepsilon_{3}$ are held constant at a value of 10 . The relative permittivity of the middle layer, $\varepsilon_{2}$, is varied from 1 to 100 . The analytical models achieve to approximate the capacitance per unit depth in close agreement with FEA results. The curves coincide closer with the simulation data for $r_{1}=0.5$ than for $r_{1}=0.1$, and start to diverge from the numerical simulation data at particularly low and high $\varepsilon_{1} / \varepsilon_{2}$-ratios. The good fit between the analytical model and FEA results is slightly biased due to the use of a logarithmic scale. Nonetheless, the analytical model shows the same trend as the simulation data and provides an appropriate estimate of the capacitance.

Lastly, the modified partial capacitance approach is verified for interdigitated electrode structures in closed configurations. Verification was performed as before, by comparing the capacitance per unit depth determined from the analytical model to the data gathered from numerical simulations. The test structures are built up of three layers, of which the bottom and top layer $\left(r_{1}=0.1, r_{3}=1.6\right.$ and $\left.r_{1}=0.5, r_{3}=1.6\right)$ have a relative permittivity of 10 , while the relative permittivity of the middle layer $\left(r_{2}=0.6\right)$ is varied from 1 to 100 . Figure 10 compares the analytical results and numerical simulation. Irrespective of the number of electrode fingers, the analytical model is able to closely fit the simulation data and approximate the capacitance of the interdigitated electrode structure.

\section{Optimization}

The models can be useful in many respects; For instance, they can be used to approximate the output of numerical simulations and physical experiments and therefore more quickly estimate how the electric field behaves in an electrochemical cell with multi-electrode arrays. Furthermore, it is possible to determine the impedance of a cell for different signal frequencies, according to $Z=-j(\omega C)^{-1}$. For media with both dielectric and conductive properties the methods described herein need to include the complex permittivity instead of only the purely dielectric permittivity [21].

Igreja \& Dias [20] proposed a strategy using analytical models for optimizing the sensitivity of interdigital capacitors. They defined a capacitance density and studied the change in capacitance as a result in a $5 \%$ change in the thickness of a sensitive layer above the electrode array. Similarly, a structure as shown in Figure 12(a) was assumed. The structure consists of an insulator, hydrophobic layer, sensitive layer, and a bulk liquid buffer. A substrate was placed below the structure. The assumed heights and permittivities of these layer are indicated in Figure 12(a). The width of the electrode fingers is fixed at $20 \mu \mathrm{m}$, which is also 
the expected minimum feature size. By using the partial capacitance approach and Schwarz-Christoffel conformal mapping (SCM) models presented, the change in capacitance as a function of a $\pm 5 \%$ change in the thickness of the sensitive layer can be determined. Figure 13 shows the results for an open configuration as a function of the metalization ratio $\eta$ and for various numbers of electrode fingers. The dotted parts of the curves do not comply with the minimum feature size constraint of $20 \mu \mathrm{m}$ or maximum array footprint of $1 \times 1 \mathrm{~mm}^{2}$. The results show that the change in the capacitance with a change in the thickness of the sensitive layer is maximum for $\eta=0.47$ and $N=24$. For closed configurations, as shown in Figure 12(b), the structure is assumed similar to the open configuration, except the buffer fluid layer is finite, and another hydrophobic layer is added. Moreover, a continuous top electrode is present. The resulting plot of $\eta$ versus the change in capacitance is shown in Figure 14. For the closed configuration the resulting optimum configuration with $w=20 \mu \mathrm{m}$ is $\eta=0.5$ and $N=24$.

Small-scale oscillations are noticeable for $\eta \approx 0.4$ in both Figure 13 and 14 . Due to the thin layers and resulting low height-to-width ratios the conformal mapping results become unstable, in which case the cell constants are approximated as $r \rightarrow 0$. For certain $\eta$ these approximations have small deviations from the otherwise well-behaved output of the models. These deviations and errors propagate through the remainder of the analysis and become particularly pronounced in evaluating the gradient of the capacitance with respect to the change in the thickness of the thin sensitive layer.

By comparing the graphs in Figure 13 and 14, a critical observation stands out. In closed configurations the change in capacitance is generally larger than in open configurations. Physically this is possibly due to the fact that the additional parallel ground plane directs the electric flux more strongly away from the bottom electrode fingers. As a result, less electric flux is trapped within the dielectric insulator and interstitial space between the electrode fingers. This observation is critical with regard to the sensitivity of insulated interdigitated electrode structures. Eliminating the need for complex geometries and material configurations to direct the electric flux to the analyte, the bottom interdigitated electrode structure can remain unchanged from that used in open configurations. The additional ground plane guides the electric flux into the sensitive region above the insulators and can potentially increase sensitivity. 


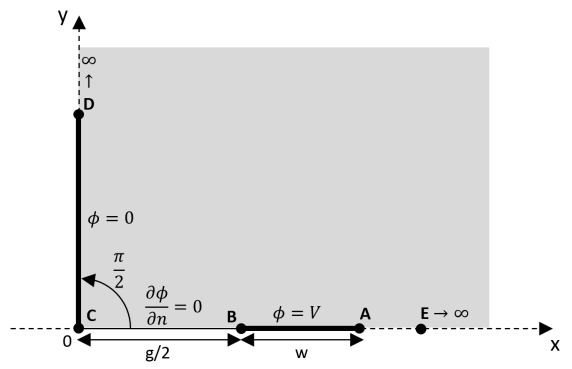

(a) Z-plane, Exterior electrodes with infinite layer

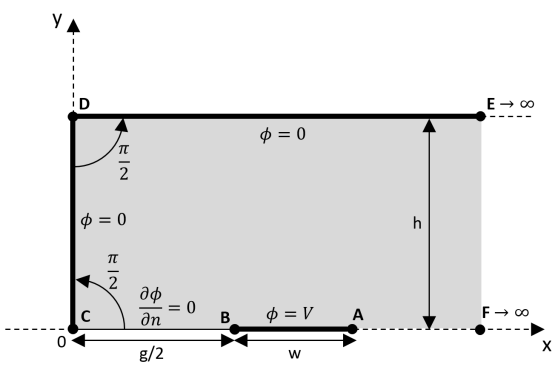

(c) Z-plane, Exterior electrodes with finite layer and Dirichlet boundary conditions

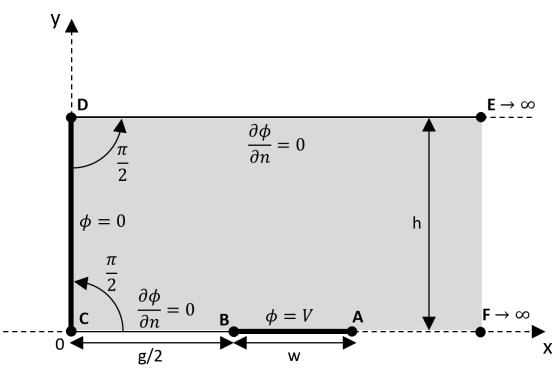

(e) Z-plane, Exterior electrodes with finite layer and Neumann boundary conditions

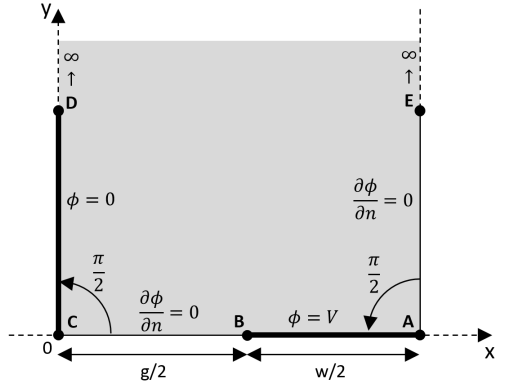

(b) Z-plane, Interior electrodes with infinite layer

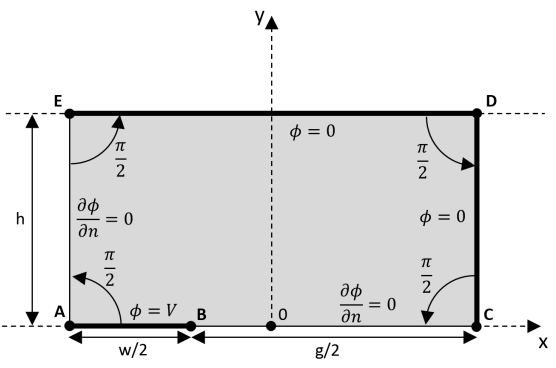

(d) Z-plane, Interior electrodes with finite layer and Dirichlet boundary conditions

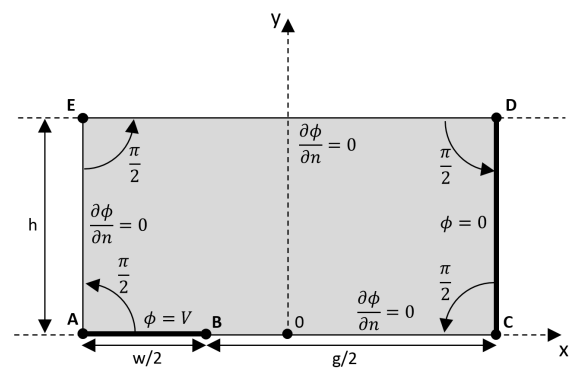

(f) Z-plane, Interior electrodes with finite layer and Neumann boundary conditions

Figure 5 Unit cells for interior and exterior electrodes in an open configuration. 


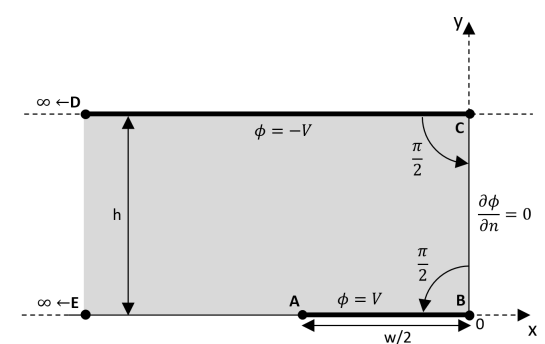

(a) Z-plane, Exterior electrodes

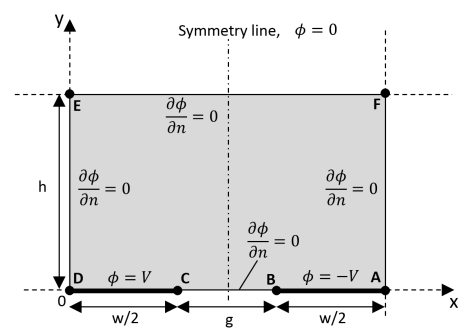

(b) Z-plane, Interior electrodes with

Neumann boundary conditions

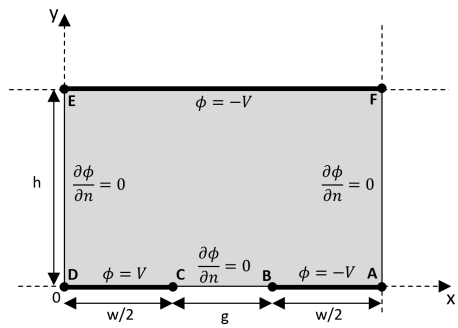

(c) Z-plane, Interior electrodes with Dirichlet boundary conditions

Figure 6 Unit cells for interior and exterior electrodes in a closed configuration.

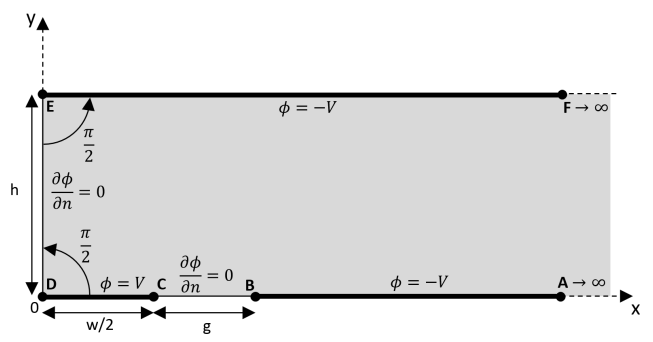

Figure 7 Z-plane, Interior electrodes with Dirichlet boundary conditions, simplified geometry.

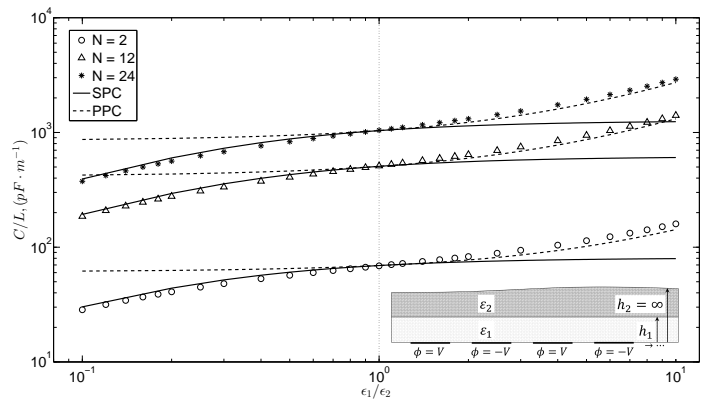

(a)

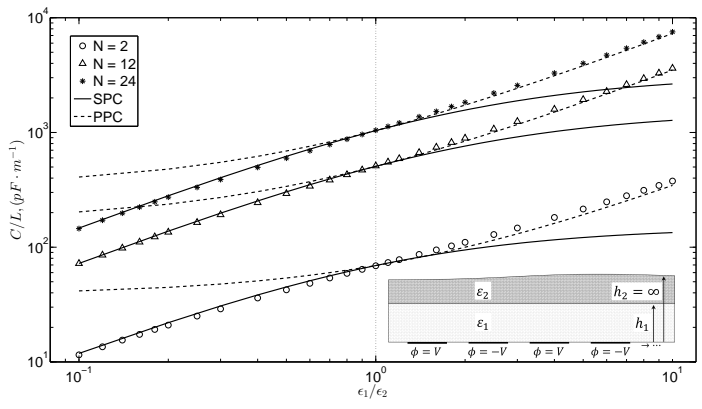

(b)

Figure 8 Capacitance per unit depth using the SPC and PPC approach for interdigitated electrode structures in open configurations and different number of electrodes $N$. The triangles indicate FE analysis results, the curves correspond to the analytical model. The inserted sketches indicate the assumed geometries. In (a), the height-to-width ratio of the bottom layer is $r_{1}=0.1 ;$ In $(b), r_{1}=0.5$. In both cases the upper layer is assumed to extend to infinity and $\eta=0.5$. The width of the electrode is fixed, $w=20$ $\mu m$. 


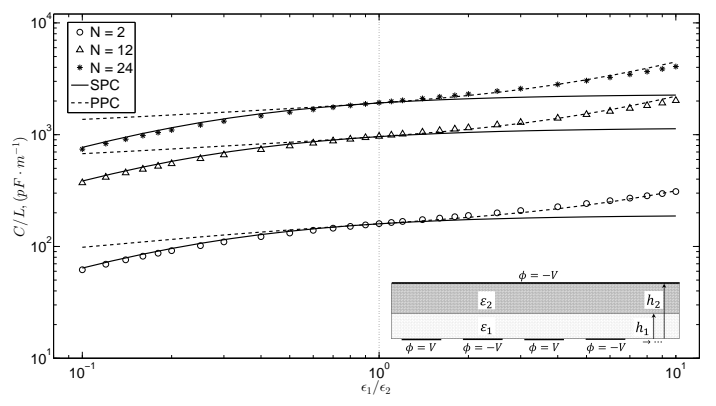

(a)

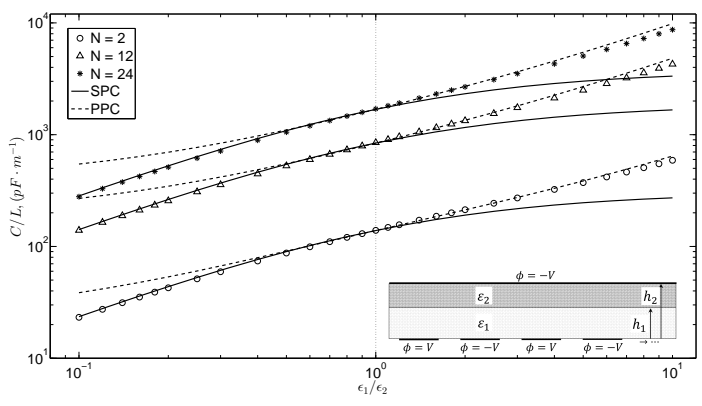

(b)

Figure 9 Capacitance per unit depth using the SPC and PPC approach for interdigitated electrode structures in closed configurations and different number of electrodes $N$. The triangles indicate FEA results, the curves correspond to the analytical model. The inserted sketches indicate the assumed geometries. In (a) $r_{1}=0.1$, and $r_{2}=1.1 ;$ In (b), $r_{1}=0.5$, and $r_{2}=1.5$. In both cases $\eta=0.5$. The width of the electrode is fixed, $w=20 \mu \mathrm{m}$.

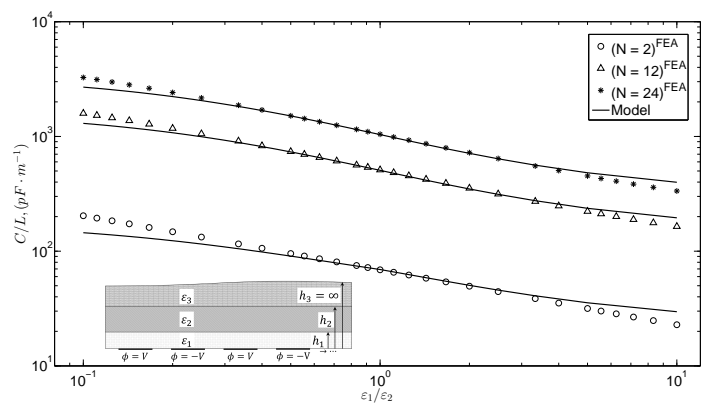

(a)

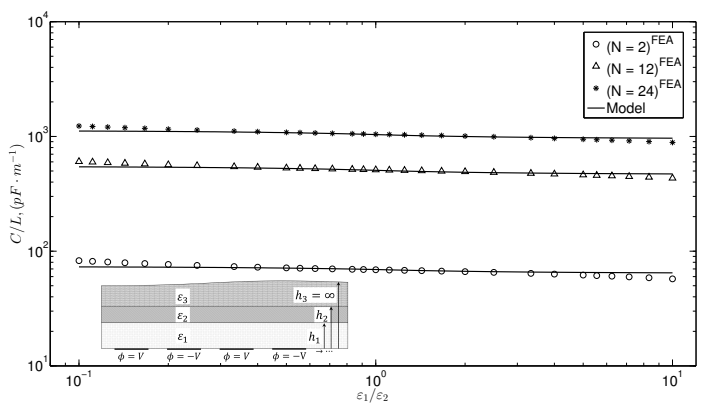

(b)

Figure 10 Capacitance per unit depth using the modified partial approach for interdigitated electrode structures in open configurations. The triangles indicate FEA results, the curves correspond to the analytical model. The inserted sketches indicate the assumed geometries. In (a), $r_{1}=0.1 ;$ In $(b), r_{1}=0.5$. In both cases $r_{2}=0.6, r_{3} \rightarrow \infty$, and $\eta=0.5$. The width of the electrode is fixed, $w=20 \mu \mathrm{m}$. 


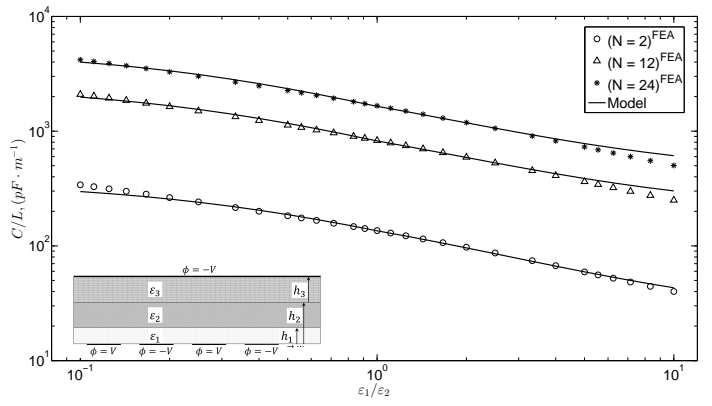

(a)

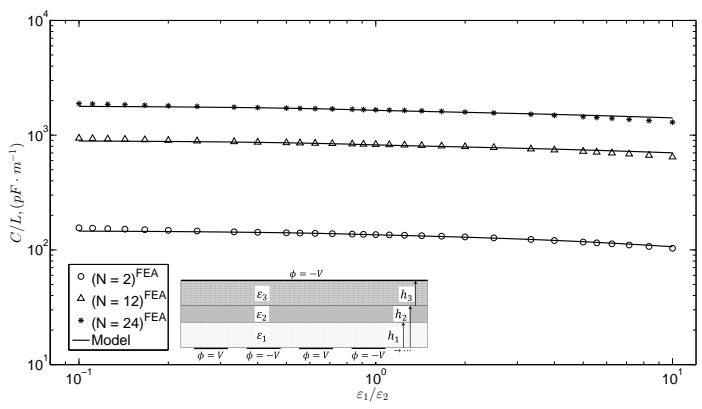

(b)

Figure 11 Capacitance per unit depth using the modified partial capacitance approach for interdigitated electrode structures in closed configurations. The triangles indicate FEA results, the curves correspond to the analytical model. The inserted sketches indicate the assumed geometries. In (a), $r_{1}=0.1$; In (b), $r_{1}=0.5$. In both cases $r_{2}=0.6, r_{3}=1.6$, and $\eta=0.5$. The width of the electrode is fixed, $w=20$ $\mu m$.

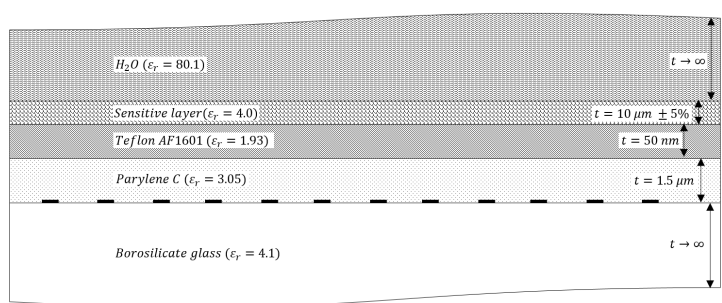

(a) Open configuration

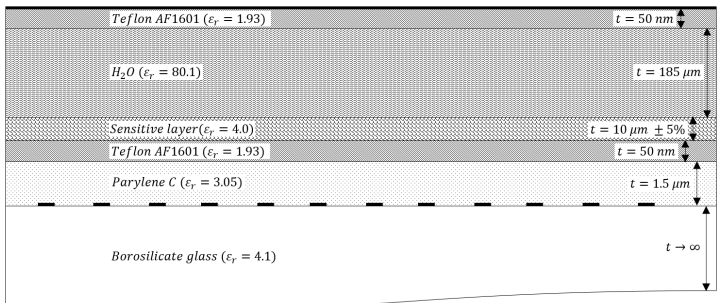

(b) Closed configuration

Figure 12 Cross section and assumed geometry of open and closed structures with a sensitive layer above the passivation layers.

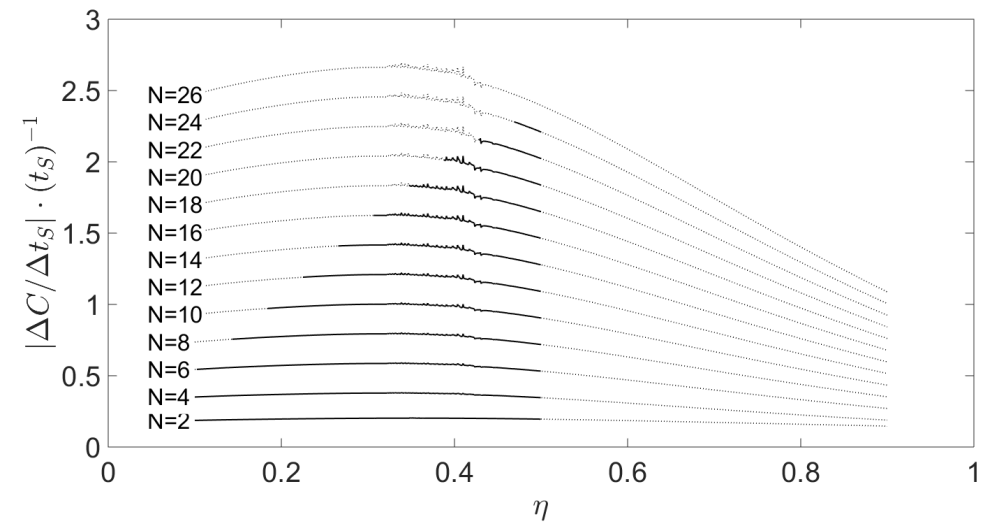

Figure 13 Change in capacitance as a result of a $5 \%$ change in the thickness of the sensitive layer $t_{S}$ in an open configuration. $\Delta C=C\left(1.05 \cdot t_{S}\right)-C\left(0.95 \cdot t_{S}\right)$. 


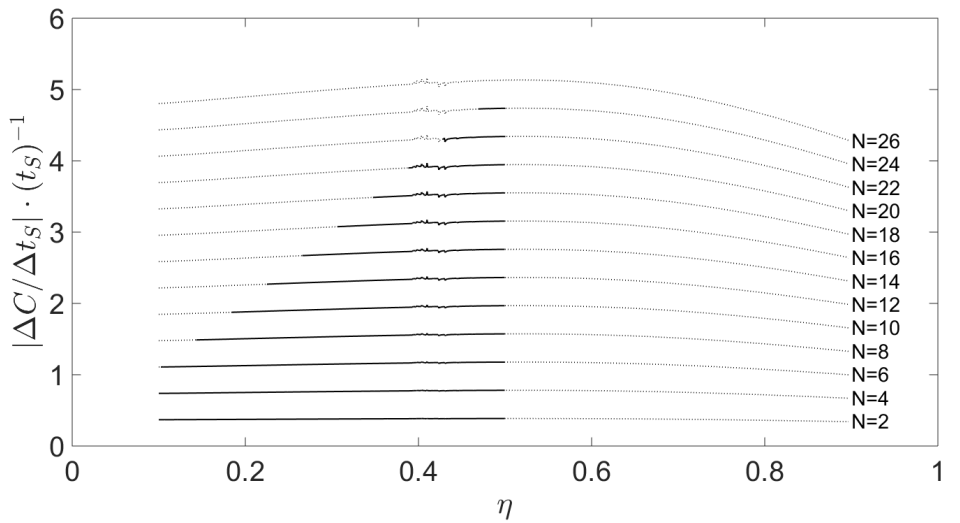

Figure 14 Change in capacitance as a result of a $5 \%$ change in the thickness of the sensitive layer $t_{S}$ in a closed configuration. $\Delta C=C\left(1.05 \cdot t_{S}\right)-C\left(0.95 \cdot t_{S}\right)$. 


\section{Conclusion}

The analytical model developed enables the capacitive characterization of interdigitated electrode structures in both open and closed configurations. Analytical expressions derived from Schwarz-Christoffel conformal transformations and an adaptation of the partial capacitance approach can determine the cell capacitance of multi-layer interdigitated electrode structures with non-monotonically varying permittivities of the layers above the electrode plane. For periodic and simple geometries, the model can be used as a design and optimization tool, replacing more complex and computationally expensive numerical simulations. As an example case, transducers can be optimized for the sensitive detection of surface effects.

\section{Acknowledgments}

The authors acknowledge CMC Microsystems for the provision of products and services that facilitated

this research, including COMSOL Multiphysics ${ }^{\circledR}$, the Natural Sciences and Engineering Research Council of Canada (NSERC) and the Ontario Centres of Excellence (OCE) for support through their granting programs. 


\section{References}

[1] A.V. Mamishev, K. Sundara-Rajan, and M. Zahn. Interdigital sensors and transducers. Proceedings of the IEEE, 92(5): 808-845, May 2004. ISSN 0018-9219. doi: 10.1109/JPROC.2004.826603. URL http://ieeexplore.iee.org/lpdocs/epic03/ wrapper.htm?arnumber $=1288505$.

[2] Junhong Min and Antje J. Baeumner. Characterization and Optimization of Interdigitated Ultramicroelectrode Arrays as Electrochemical Biosensor Transducers. Electroanalysis, 16(9):724-729, May 2004. ISSN 1040-0397. doi: 10.1002/elan. 200302872. URL http://doi.wiley.com/10.1002/elan.200302872.

[3] Zhiwei Zou, Junhai Kai, Michael J. Rust, Jungyoup Han, and Chong H. Ahn. Functionalized nano interdigitated electrodes arrays on polymer with integrated microfluidics for direct bio-affinity sensing using impedimetric measurement. Sensors and Actuators A: Physical, 136(2):518-526, May 2007. ISSN 09244247. doi: 10.1016/j.sna.2006.12.006. URL http://linkinghub.elsevier.com/retrieve/pii/S0924424706007588.

[4] Madhukar Varshney and Yanbin Li. Interdigitated array microelectrodes based impedance biosensors for detection of bacterial cells. Biosensors $\&$ bioelectronics, 24(10):2951-60, June 2009. ISSN 1873-4235. doi: 10.1016/j.bios.2008.10.001. URL http://www.ncbi.nlm.nih.gov/pubmed/19041235.

[5] Kosuke Ino, Yusuke Kitagawa, Tsuyoshi Watanabe, Hitoshi Shiku, Masahiro Koide, Tomoaki Itayama, Tomoyuki Yasukawa, and Tomokazu Matsue. Detection of hormone active chemicals using genetically engineered yeast cells and microfluidic devices with interdigitated array electrodes. Electrophoresis, 30(19):3406-12, October 2009. ISSN 1522-2683. doi: 10.1002/elps.200900244. URL http://www.ncbi.nlm.nih.gov/pubmed/19802852.

[6] Gerald M Birnbaumer, Peter a Lieberzeit, Lukas Richter, Romana Schirhagl, Marcus Milnera, Franz L Dickert, Andrew Bailey, and Peter Ertl. Detection of viruses with molecularly imprinted polymers integrated on a microfluidic biochip using contact-less dielectric microsensors. Lab on a chip, 9(24):3549-56, December 2009. ISSN 1473-0197. doi: 10.1039/b914738a. URL http://www.ncbi.nlm.nih.gov/pubmed/20024035.

[7] Christian Jungreuthmayer, Gerald M Birnbaumer, Juergen Zanghellini, and Peter Ertl. 3D numerical simulation of a labon-a-chip-increasing measurement sensitivity of interdigitated capacitors by passivation optimization. Lab on a chip, 11 (7):1318-25, April 2011. ISSN 1473-0189. doi: 10.1039/c0lc00543f. URL http://www.ncbi.nlm.nih.gov/pubmed/21331426.

[8] Haibo Li and Rashid Bashir. Dielectrophoretic separation and manipulation of live and heat-treated cells of Listeria on microfabricated devices with interdigitated electrodes. Sensors and Actuators B: Chemical, 86:215-221, 2002. URL http://www.sciencedirect.com/science/article/pii/S0925400502001727.

[9] Liju Yang, Padmapriya P Banada, Mohammad R Chatni, Kwan Seop Lim, Arun K Bhunia, Michael Ladisch, and Rashid Bashir. A multifunctional micro-fluidic system for dielectrophoretic concentration coupled with immuno-capture of low numbers of Listeria monocytogenes. Lab on a chip, 6(7):896-905, July 2006. ISSN 1473-0197. doi: 10.1039/b607061m. URL http://www.ncbi.nlm.nih.gov/pubmed/16804594. 
[10] D.F. Chen, H. Du, and W.H. Li. Bioparticle separation and manipulation using dielectrophoresis. Sensors and Actuators A: Physical, 133(2):329-334, February 2007. ISSN 09244247. doi: 10.1016/j.sna.2006.06.029. URL http: //linkinghub.elsevier.com/retrieve/pii/S0924424706004377.

[11] Hui-Sung Moon, Hee Taek Im, Ahmi Choi, and Hyo-Il Jung. Real-time detection of food-borne bacterial adenosine triphosphate (ATP) using dielectrophoretic force and a bioluminescence sensor. Microchimica Acta, 170(3-4):283-288, May 2010. ISSN 0026-3672. doi: 10.1007/s00604-010-0370-9. URL http://link.springer.com/10.1007/s00604-010-0370-9.

[12] Khashayar Khoshmanesh, Saeid Nahavandi, Sara Baratchi, Arnan Mitchell, and Kourosh Kalantar-zadeh. Dielectrophoretic platforms for bio-microfluidic systems. Biosensors \& bioelectronics, 26(5):1800-14, January 2011. ISSN $1873-4235$. doi: 10.1016/j.bios.2010.09.022. URL http://www.ncbi.nlm.nih.gov/pubmed/20933384.

[13] Roland Schinzinger. Conformal Mapping: Methods and Applications. Elsevier Science Publishers B.V., Amsterdam, 1991. ISBN 0-444-88806-3.

[14] W Olthuis, W Streekstra, and P Bergveld. Theoretical and experimental determination of cell constants of planarinterdigitated electrolyte conductivity sensors. Sensors and Actuators B: Chemical, 25:252-256, 1995. URL http: //www.sciencedirect.com/science/article/pii/0925400595850538.

[15] Björan Timmer, Wouter Sparreboom, Wouter Olthuis, Piet Bergveld, and Albert van den Berg. Optimization of an electrolyte conductivity detector for measuring low ion concentrations. Lab on a chip, 2(2):121-4, May 2002. ISSN 1473-0197. doi: 10.1039/b201225a. URL http://www.ncbi.nlm.nih.gov/pubmed/15100845.

[16] Jongin Hong, Dae Sung Yoon, Sung Kwan Kim, Tae Song Kim, Sanghyo Kim, Eugene Y. Pak, and Kwangsoo No. AC frequency characteristics of coplanar impedance sensors as design parameters. Lab on a chip, 5(3):270-9, March 2005. ISSN 1473-0197. doi: 10.1039/b410325d. URL http://www.ncbi.nlm.nih.gov/pubmed/15726203.

[17] Pontus Linderholm and Philippe Renaud. Comment on "AC frequency characteristics of coplanar impedance sensors as design parameters" by Jongin Hong, Dae Sung Yoon, Sung Kwan Kim, Tae Song Kim, Sanghyo Kim, Eugene Y. Pak and Kwangsoo No, Lab Chip, 2005, 5, 270. Lab on a chip, 5(12):1416-7; author reply 1418, December 2005. ISSN $1473-0197$. doi: 10.1039/b512077b. URL http://www.ncbi.nlm.nih.gov/pubmed/16286976.

[18] Peter Van Gerwen, Wim Laureyn, Wim Laureys, Guido Huyberechts, Maaike Op De Beeck, Kris Baert, Jan Suls, Willy Sansen, P. Jacobs, Lou Hermans, and Robert Mertens. Nanoscaled interdigitated electrode arrays for biochemical sensors. Sensors and Actuators B: Chemical, 49:73-80, 1998.

[19] T Sun, N G Green, S Gawad, and H Morgan. Analytical electric field and sensitivity analysis for two microfluidic impedance cytometer designs. IET nanobiotechnology / IET, 1(5):69-79, October 2007. ISSN 1751-8741. doi: 10.1049/iet-nbt: 20070019. URL http://www.ncbi.nlm.nih.gov/pubmed/17764376.

[20] Rui Igreja and C.J. Dias. Analytical evaluation of the interdigital electrodes capacitance for a multi-layered structure. Sensors and Actuators A: Physical, 112(2-3):291-301, May 2004. ISSN 09244247. doi: 10.1016/j.sna.2004.01.040. URL http://linkinghub.elsevier.com/retrieve/pii/S0924424704000779. 
[21] Rui Igreja and C.J. Dias. Extension to the analytical model of the interdigital electrodes capacitance for a multi-layered structure. Sensors and Actuators A: Physical, 172(2):392-399, December 2011. ISSN 09244247. doi: 10.1016/j.sna.2011. 09.033. URL http://linkinghub.elsevier.com/retrieve/pii/S0924424711005474.

[22] Huey-daw Wu, Zhihang Zhang, Frank Barnes, Charles M. Jackson, Aron Kain, and J. D. Cuchiaro. Voltage Tunable Capacitors Using High Temperature Superconductors and Ferroelectrics. IEEE Transactions on Applied Superconductivity, 4(3):156-160, September 1994.

[23] Spartak S. Gevorgian, Torsten Martinsson, Peter L. J. Linner, and Erik Ludvig Kollberg. CAD Models for Multilayered Substrate Interdigital Capacitors. IEEE Transactions on Microwave Theory and Techniques, 44(6):896-904, June 1996.

[24] R. B. Fair. Digital microfluidics: is a true lab-on-a-chip possible? Microfluidics and Nanofluidics, 3(3):245-281, March 2007. ISSN 1613-4982. doi: 10.1007/s10404-007-0161-8. URL http://link.springer.com/10.1007/s10404-007-0161-8.

[25] Kihwan Choi, Alphonsus H C Ng, Ryan Fobel, and Aaron R Wheeler. Digital microfluidics. Annual review of analytical chemistry, 5:413-40, January 2012. ISSN 1936-1335. doi: 10.1146/annurev-anchem-062011-143028. URL http://www.ncbi.nlm.nih.gov/pubmed/22524226.

[26] Mais J Jebrail, Michael S Bartsch, and Kamlesh D Patel. Digital microfluidics: a versatile tool for applications in chemistry, biology and medicine. Lab on a chip, 12(14):2452-63, July 2012. ISSN 1473-0189. doi: 10.1039/c2lc40318h. URL http://www.ncbi.nlm.nih.gov/pubmed/22699371.

[27] Wyatt C Nelson and Chang-Jin Kim. Droplet Actuation by Electrowetting-on-Dielectric (EWOD): A Review. Journal of Adhesion Science and Technology, 26:1747-1771, 2012.

[28] Matthew Gillick, Ian D Robertson, and Jai S Joshi. An Analytical Method for Direct Calculation of. IEEE Transactions on Microwave Theory and Techniques, 41(9):1606-1610, September 1993.

[29] C. Veyres and V . Fouad Hanna. Extension of the application of conformal mapping techniques to coplanar lines with finite dimensions. International Journal of Electronics, 48(1):47-56, 1980.

[30] Giovanni Ghione and Michele Goano. Revisiting the Partial-Capacitance Approach to the Analysis of Coplanar Transmission Lines on Multilayered Substrates. IEEE Transactions on Microwave Theory and Techniques, 51(9): 2007-2014, 2003.

[31] Spartak S. Gevorgian. Basic characteristics of two layered substrate coplanar waveguides. Electronics Letters, 30(15): 1236-1237, July 1994.

[32] Ning Hua Zhu and Edwin Yue Bun Pun. Analytical formulas for calculating the effective dielectric constants of coplanar lines for OIC applications. Microwave and Optical Technology Letters, 9(4):229-232, July 1995.

[33] Giovanni Ghione, Senior Member, Michele Goano, Gianluigi Madonna, Student Member, Guido Omegna, Marco Pirola, Sergio Bosso, Davide Frassati, and Aldo Perasso. Microwave Modeling and Characterization of Thick Coplanar Waveguides on Oxide-Coated Lithium Niobate Substrates for Electrooptical Applications. IEEE Transactions on Microwave Theory and Techniques, 47(12):2287-2293, December 1999. 
[34] Roland Schinzinger. Conformal Mapping: Methods and Applications. Elsevier Science Publishers B.V., Amsterdam, 1991. ISBN 0-444-88806-3.

[35] Tao Sun, Hywel Morgan, and Nicolas Green. Analytical solutions of ac electrokinetics in interdigitated electrode arrays: Electric field, dielectrophoretic and traveling-wave dielectrophoretic forces. Physical Review E, 76(4):046610, October 2007. ISSN 1539-3755. doi: 10.1103/PhysRevE.76.046610. URL http://link.aps.org/doi/10.1103/PhysRevE.76.046610.

[36] Steffen Otto Peter Blume. A Multi-Electrode Array for Impedance Spectroscopy in a Digital Microfluidic Device. M.A.Sc. thesis, University of Toronto, 2014.

[37] Tobin A. Driscoll and Lloyd N. Trefethen. Schwarz-Christoffel mapping. Cambridge University Press, Cambridge, UK, 2002. ISBN 0-521-80726-3.

[38] Wolfram Luther and Werner Otten. Reliable Computation of Elliptic Functions. Journal of Universal Computer Science, 4(1):25-33, January 1998.

[39] Steffen Otto Peter Blume, Michael J. Schertzer, Ridha Ben-Mrad, and Pierre E. Sullivan. Analytical models to determine the electric field characteristics of a multi-electrode impedimetric immunosensor in a digital microfluidic device. In ASME 2014 International Mechanical Engineering Congress \& Exposition, pages IMECE2014-37571, Montreal, Quebec, Canada, November 2014. 